DAMTP-R/94/15

March, 1994

\title{
DIVERGENCES IN THE MODULI SPACE INTEGRAL AND ACCUMULATING HANDLES IN THE INFINITE-GENUS LIMIT
}

\author{
Simon Davis \\ International Centre for Theoretical Physics \\ Strada Costiera 11, 34100 Trieste, Italy \\ and \\ Department of Applied Mathematics and Theoretical Physics \\ University of Cambridge \\ Silver Street, Cambridge CB3 9EW *
}

\begin{abstract}
The symmetries associated with the closed bosonic string partition function are examined so that the integration region in Teichmuller space can be determined. The conditions on the period matrix defining the fundamental region can be translated to relations on the parameters of the uniformizing Schottky group. The growth of the lower bound for the regularized partition function is derived through integration over a subset of the fundamental region.
\end{abstract}

* Present address 


\section{Introduction}

The elimination of infrared divergences in scattering amplitudes of superstring theories promises a consistent quantum theory including gravity as part of the low-energy limit. An understanding of space-time at the most fundamental level could be achieved with the development of such a theory.

This requires a complete formulation of the theory based on the sum over string histories arising in the path integral. Both the entire perturbative amplitude, and possibly nonperturbative effects, could be obtained in this approach.

Interest has centered recently on a divergence in bosonic string theory that arises from summing the contributions at each loop, which have been rendered finite individually through a genus-independent regularization [1]. The cut-off introduced for the bosonic string excludes effectively closed surfaces of sufficiently large genus, but it will be shown that surfaces having an ideal boundary with positive linear measure do arise in the sum over all orders in the perturbation expansion. The source of the divergence can then be traced to these surfaces, which may be interpreted as representing a non-perturbative effect in string theory.

The counting of these surfaces, and the effectively closed surfaces at higher genus, in the path integral could impact on the finiteness properties of the superstring path integral.

The investigation of the divergence begins here with a study of the measure and the domain in the integral for the partition function. The measure is derived from the path integral weighting factor and a choice of coordinates on the the moduli space of metrics. The two most frequently used measures are those defined by the light-cone and Polyakov approaches, which require manifest unitarity or covariance of the string theory respectively. The light-cone diagram is constructed so that the momentum of the external string is proportional to the distance between the cuts at initial and final times, while the internal cuts correspond to the joining and splitting of strings. The conformal mapping from the string diagram to a planar domain with disks removed and punctures at the positions of the 
vertex operators transforms paths from the boundary of the diagram to the internal cuts and the paths around the cuts to a-cycles and b-cycles respectively [2]. The planar domains with $2 \mathrm{~g}$ disks removed are the Schottky covering surfaces for a Riemann surface of genus g, and by the retrosection theorem, every compact Riemann surface can be uniformized by a Schottky group generated by g Mobius transformations [3].

The path integral in the Euclidean formulation is

$$
\sum_{g=0}^{\infty} \kappa^{g} \int \frac{D\left[h_{\alpha \beta}\right] D\left[X^{\mu}\right]}{\operatorname{Vol}\left(\operatorname{Diff} \Sigma_{g}\right) \operatorname{Vol}\left(\operatorname{Conf} \Sigma_{g}\right)} e^{-\frac{1}{2} \int_{\Sigma_{g}} d^{2} \xi \sqrt{h} h^{\alpha \beta} \partial_{\alpha} X^{\mu} \partial_{\beta} X_{\mu}}
$$

The integration over the space of metrics is reduced first to the $3 \mathrm{~g}-3$ complex-dimensional Teichmuller space $T_{g}$ by factoring by the identity component of the diffeomorphism group Dif $f_{0}\left(\Sigma_{g}\right)$, and then it can be reduced further to the moduli space $M_{g}$ by factoring by the mapping class group $\operatorname{Diff}\left(\Sigma_{g}\right) / \operatorname{Dif} f_{0}\left(\Sigma_{g}\right)$. Since a compact Riemann surface of genus g can be represented as $D / \Gamma$, where $\Gamma$ is a Schottky group with g generators and $\mathrm{D}$ is the set of ordinary points of $\Gamma$ [the complement of the set of limit points in the extended complex plane], the space of inequivalent complex structures on the surface can be coordinatized by the multipliers $K_{n}, n=1, \ldots, g$ and fixed points $\xi_{1 n}, \xi_{2 n}, n=1, \ldots, g$ of the generating Mobius transformations $T_{1}, \ldots, T_{g}$, with three of the parameters fixed by an overall $\operatorname{PSL}(2, \mathbb{C})$ transformation.

The measure on moduli space can then be expressed [4] as

$$
\begin{array}{r}
\prod_{m=1}^{g} \frac{d^{2} K_{m}}{\left|K_{m}\right|^{4}}\left|1-K_{m}\right|^{4} \frac{1}{\operatorname{Vol}(S L(2, \mathbb{C}))} \prod_{n=1}^{g} \frac{d^{2} \xi_{1 n} d^{2} \xi_{2 n}}{\left|\xi_{1 n}-\xi_{2 n}\right|^{4}}[\operatorname{det}(\operatorname{Im} \tau)]^{-13 g} \\
\prod_{\alpha}^{\prime} \prod_{p=1}^{\infty}\left|1-K_{\alpha}^{p}\right|^{-48} \prod_{\alpha}^{\prime}\left|1-K_{\alpha}\right|^{-4}
\end{array}
$$

where $\prod_{\alpha}^{\prime}$ is a product over conjugacy classes of primitive elements of the group $\Gamma$ and $\tau$ is the period matrix [5]

$$
\tau_{m n}=\frac{1}{2 \pi i}\left[\ln K_{m} \delta_{m n}+\sum_{\alpha}{ }^{(m, n)} \ln \left(\frac{\xi_{1 m}-V_{\alpha} \xi_{1 n}}{\xi_{1 m}-V_{\alpha} \xi_{2 n}} \frac{\xi_{2 m}-V_{\alpha} \xi_{2 n}}{\xi_{2 m}-V_{\alpha} \xi_{1 n}}\right)\right]
$$


with $\sum_{\alpha}^{(m, n)}$ excluding elements with $T_{m}^{ \pm 1}$ as the left-most member and $T_{n}^{ \pm 1}$ as the rightmost member.

An alternative expression for the partition function can be obtained through the evaluation of the determinant factors giving rise to Selberg trace functions. The equivalence of the two approaches has been demonstrated by relating the determinants of these operators and path integrals over the positions of interaction points [6]. As the equivalence should extend to measures, the differential volume element in equation (2) can be used to study the behaviour of the partition function with respect to the genus. Moreover, the Schottky group formalism has been found to be one of the few methods useful for calculating physically relevant higher-genus scattering amplitudes [7][8].

It has been established that the string integrand is invariant with respect to the symplectic modular group [9], which is associated with a change of homology basis. When the a-cycles and b-cycles transform as

$$
\left(\begin{array}{l}
a^{\prime} \\
b^{\prime}
\end{array}\right)=\left(\begin{array}{ll}
A & B \\
C & D
\end{array}\right)\left(\begin{array}{l}
a \\
b
\end{array}\right) \quad\left(\begin{array}{ll}
A & B \\
C & D
\end{array}\right) \in S p(2 g ; \mathbb{Z})
$$

the canonical basis of one-forms transforms as

$$
\left[\tilde{\omega}_{1}, \ldots, \tilde{\omega}_{g}\right]=\left[\omega_{1}, \ldots, \omega_{g}\right](A+B \tau)^{-1}
$$

and the period matrix transforms as

$$
\tau \rightarrow(C+D \tau)(A+B \tau)^{-1}
$$

The transformations are induced by diffeomorphisms not connected to the identity. A particular set of mappings, Dehn twists, are defined by cutting out cylinders in the surface, twisting the cylinder around a chosen cycle, and gluing the cylinder back to the surface [10]. These twists mix the a-cycles and b-cycles as given in equation (4) and may generate transformations that are not connected to the identity compoment of the diffeomorphism group. As the integer entries of the symplectic matrix $M$ are restricted by the condition that the determinant should equal 1 and that the intersection matrix is preserved $M^{T} J M=J$, 
the image of the symplectic group in the mapping class group must be supplemented by the transformations not acting on the homology basis, the Torelli group [11].

Integration over the Schottky group parameters, or equivalently the period matrix elements, through equation (3), already implies factorization by the Torelli group. An integration region in the space of period matrices can be defined using the action of the symplectic modular group in equation (6). Transformations in the Schottky group space $\left\{K_{n}, \xi_{1 n}, \xi_{2 n}\right\}$ also lead to a mixing of a- and b- cycles, because configurations in which the isometric circles of the fundamental generators are tangent allow for paths which are combinations of the original a- and b-cycles. Thus, an integral over a region in parameter space is only equivalent to a lower bound for the moduli space integral when the region lies inside the fundamental domain of the modular group. The estimate of the growth of the bosonic string partition function based on an integral over this subdomain may be sufficient, because the measure is positive on all of moduli space, as a result of the positivedefiniteness of imaginary part of the period matrix and the feasibility of expressing the rest of the measure as the squared modulus of a holomorphic section of a line bundle over moduli space [12][13].

\section{Configurations of Isometric Circles and Bounds for the Regularized Integral}

There is a one-to-one correspondence between points in the fundamental domain of the uniformizing Schottky group $\Gamma$ and the Riemann surface [14]. The identification of points on the handle with points in the fundamental domain can be achieved by identifying specific circles outside the isometric circles, representing the boundaries of the removed disks in $\hat{\mathbb{C}}$, with boundaries of the bases of handles and joining the isometric circles at the middle of the handle. About each handle, there exists a closed curve which is geodesic. The genus-independent cut-off on the length of closed geodesics can be translated, therefore, to constraints on the sizes of the isometric circles.

For $g \geq 2$, the universal covering surface of the Riemann surface is the Poincare disk or the upper-half plane with a hyperbolic metric, that can be projected onto an intrinsic metric with constant curvature $R=-1$. The restriction to $R=-1$ metrics defines a slice in 
the space of metrics, which is necessary in the reduction of the Polyakov path integral to a moduli space integral [15], and it implies that the area increases linearly with the genus.

Now consider the stereographic projection from $\hat{\mathbb{C}}$ to the sphere. The Riemann surfaces can be constructed directly by joining the isometric circles to create handles. Since the stereographic projection is genus-independent, the dimensions of the sphere in the target space will remain unchanged as the genus increases, but the dilation of the areas, and therefore the distances, in the intrinsic metric on the sphere, with $g$ handles, forces a decrease in the size of the isometric circles, the spherical condition $\left|\gamma_{n}\right|^{-2} \sim \frac{1}{g}$ is required for consistency with the cut-off on closed geodesic lengths. Moreover, the relation,

$$
\frac{\left|\gamma_{n}\right|^{-2}}{\left|\xi_{1 n}-\xi_{2 n}\right|^{2}}=\frac{\left|K_{n}\right|}{\left|1-K_{n}\right|^{2}}
$$

can be used to translate this condition to constraints on the multipliers and fixed points.

As the relation (7) holds for transformations on the extended complex plane, the mapping of disks on $\hat{\mathbb{C}}$ to the sphere must be used before placing restrictions on the multipliers and fixed points. If the isometric circles are confined to a bounded region in $\hat{\mathbb{C}}$, the genusdependence of $\left|\gamma_{n}\right|^{-1}$ will be unaltered by the stereographic projection. There are three categories of isometric circles consistent with the condition $\left|\gamma_{n}\right|^{-2} \sim \frac{1}{g}$ in a bounded region in $\hat{\mathbb{C}}$.

$$
\begin{array}{ll}
\text { (i) } \frac{\epsilon_{0}}{g} \leq\left|K_{n}\right| \leq \frac{\epsilon_{0}^{\prime}}{g} & \delta_{0} \leq\left|\xi_{1 n}-\xi_{2 n}\right| \leq \delta_{0}^{\prime} \\
\text { (ii) } \frac{\epsilon_{0}}{g^{1-2 q}} \leq\left|K_{n}\right| \leq \frac{\epsilon_{0}^{\prime}}{g^{1-2 q}} & \frac{\delta_{0}}{g^{q}} \leq\left|\xi_{1 n}-\xi_{2 n}\right| \leq \frac{\delta_{0}^{\prime}}{g^{q}} \quad 0<q<\frac{1}{2} \\
\text { (iii) } \epsilon_{0} \leq\left|K_{n}\right| \leq \epsilon_{0}^{\prime} & \frac{\delta_{0}}{\sqrt{g}} \leq\left|\xi_{1 n}-\xi_{2 n}\right| \leq \frac{\delta_{0}^{\prime}}{\sqrt{g}}
\end{array}
$$

For the first category of configurations, lower bounds can be placed on the primitiveelement products and determinant factor in equation (2). Since

$$
\begin{aligned}
\prod_{\alpha}{ }^{\prime}\left|1-K_{\alpha}\right|^{-1} & >\exp \left(-\sum_{\alpha}{ }^{\prime}\left|K_{\alpha}\right|\right) \\
\sum_{\alpha}{ }^{\prime}\left|K_{\alpha}\right| & <\sum_{n_{l}} \frac{\left|\gamma_{n_{l}}\right|^{-2}}{\left|\xi_{1 n_{l}}+\frac{\delta_{n_{l}}}{\gamma_{n_{l}}}\right|^{2}}+\sum_{n_{l}} \frac{\left|\gamma_{n_{l}}\right|^{-2}}{\left|\xi_{2 n_{l}}-\frac{\alpha_{n_{l}}}{\gamma_{n_{l}}}\right|^{2}}+\sum_{V_{\tilde{\alpha}}=T_{n_{l}} V_{\beta}} \frac{\left|\gamma_{\tilde{\alpha}}\right|^{-2}}{\left|\xi_{1 \tilde{\alpha}}+\frac{\delta_{\tilde{\alpha}}}{\gamma_{\tilde{\alpha}}}\right|^{2}}
\end{aligned}
$$


it suffices to estimate the growth of the bounds of these sums. As $\left|\gamma_{n_{l}}\right|^{-2}<\frac{\epsilon_{0}^{\prime}}{\left[1-\frac{\epsilon_{0}^{\prime}}{g}\right]^{2}} \frac{\delta_{0}^{\prime 2}}{g}$, the sum over the fundamental generators $\left\{I_{T_{n_{l}}^{ \pm 1}}\right\}$ is less than $2 \frac{\epsilon_{0}^{\prime}}{\left[1-\frac{\epsilon_{0}^{\prime}}{g}\right]^{2}} \frac{\delta_{0}^{\prime 2}}{\delta_{0}^{2}}$. The terms in the remaining sum can be grouped according to the number of generators in $V_{\tilde{\alpha}}$. When the product consists of two elements, $V_{\tilde{\alpha}}=T_{n_{l_{1}}} T_{n_{l_{2}}}$, and

$$
\left|\gamma_{\tilde{\alpha}}\right|^{-2}=\left|\gamma_{n_{l_{1}}}\right|^{-2}\left|\gamma_{n_{l_{2}}}\right|^{-2}\left|\frac{\delta_{n_{l_{1}}}}{\gamma_{n_{l_{1}}}}+\frac{\alpha_{n_{l_{2}}}}{\gamma_{n_{l_{2}}}}\right|^{-2} \leq \frac{\epsilon_{0}^{\prime 2}}{\left[1-\frac{\epsilon_{0}^{\prime}}{g}\right]^{4}} \frac{\delta_{0}^{\prime 4}}{g^{2}}\left|\frac{\delta_{n_{l_{1}}}}{\gamma_{n_{l_{1}}}}+\frac{\alpha_{n_{l_{2}}}}{\gamma_{n_{l_{2}}}}\right|^{-2}
$$

Now consider a dense packing of isometric circles spaced apart by a distance of $O\left(\frac{1}{\sqrt{g}}\right)$ in a finite region of the complex plane. In a hexagonal arrangement of $2 \mathrm{~g}$ circles, the circles can be indexed by the level number 1 , defined by their distance from the origin, the number of circles at level $l$ is $6(1-1)$, and the number of levels $\left[\frac{1}{2}+\frac{1}{6} \sqrt{9+24 g}\right]$.

The level numbers of $I_{T_{n_{l_{1}}}}$ and $I_{T_{n_{l_{2}}}^{-1}}$ can be chosen to be $l$ and $l+l_{0}$ respectively. Indexing the circles at these levels by $j_{1}=0,1, \ldots, 6(l-1)-1$ and $j_{2}=0,1, \ldots, 6\left(l+l_{0}-1\right)-1$, it follows that

$$
\left|\frac{\delta_{T_{n_{l_{1}}^{(j)}}^{\left(j_{1}\right)}}}{\gamma_{T_{n_{l_{1}}}^{\left(j_{1}\right)}}}+\frac{\alpha_{T_{n_{l_{2}}}^{\left(j_{2}\right)}}}{\gamma_{T_{n_{l_{2}}}^{\left(j_{2}\right)}}}\right|^{-2} \leq\left[d_{l+l_{0}}^{2}+d_{l}^{2}-2 d_{l} d_{l+l_{0}} \cos \left(\frac{i\left(j_{1}, j_{2}\right) \pi}{3\left(l+l_{0}-1\right)}\right)\right]^{-1}
$$

where $d_{l}$ is the distance from a level $l$ circle to the center of the configuration and $i\left(j_{1}, j_{2}\right)$ takes integer values in the range $\left[0,6\left(l+l_{0}-1\right)-1\right]$. Since the distance between the fixed points is greater than $\delta_{0}$, there is no correlation between the distances $d\left(I_{T_{n_{l_{1}, j_{1}}}}, I_{T_{n_{l_{2}, j_{2}}}^{-1}}\right)$ and $d\left(I_{T_{n_{l_{1}, j_{1}}}^{-1}}, I_{T_{n_{l_{2}, j_{2}}}}\right)$. A lower bound for the latter distance can be obtained through the densest packing of $6\left(l+l_{0}-1\right)$ circles $\left\{I_{T_{n_{l_{2}}}}\right\}$ about $I_{T_{n_{l_{1}, j_{1}}}^{-1}}$. As the average value of $\left[d\left(I_{T_{n_{l_{1}, j_{1}}}^{-1}},\left\{I_{T_{n_{l_{2}, j_{2}}}}\right\}\right]^{-2}\right.$ in this packing is about $\frac{g}{2 \delta_{0}^{2}} \frac{\ln \left(l+l_{0}-1\right)}{l+l_{0}-1}$, the sum over the elements $V_{\tilde{\alpha}}=T_{n_{l_{1}}}^{ \pm 1} T_{n_{l_{2}}}^{ \pm 1}$ in $\sum_{\alpha}{ }^{\prime}\left|K_{\alpha}\right|$ is less than

$$
\begin{aligned}
& \epsilon_{0}^{\prime 2}\left[1-\frac{\epsilon_{0}^{\prime}}{g}\right]^{-4} \frac{\delta_{0}^{\prime 4}}{\delta_{0}^{4}} \\
& \quad \cdot \sum_{l=2}^{\left[\frac{1}{2}+\frac{1}{6} \sqrt{9+24 g}\right]} 18[l-1]\left[1+\frac{1}{2 l-2}+\frac{1}{2} \sum_{\substack{l_{0}=-l+2 \\
l_{0} \neq 0}}^{\left[\frac{1}{2}+\frac{1}{6} \sqrt{9+24 g}-l\right.} \frac{\ln \left(l+l_{0}-1\right)}{l+l_{0}-1}\left|\frac{1}{l_{0}}+\frac{1}{2 l+l_{0}-2}\right|\right]
\end{aligned}
$$


This bound increases linearly with respect to the genus, a property which continues to hold for the higher-order terms, since summation over the elements $V_{\tilde{\alpha}}=T_{n_{l_{1}}}^{ \pm 1} \ldots T_{n_{l_{r}}}^{ \pm 1}$ introduces r-1 factors of the form $6\left[l^{T_{n_{l}}^{ \pm 1}}-1\right]$ producing a term of order $O\left(g^{r-1}\right)$, while iteration of the formula (9) leads to a term of order $O\left(g^{-(r-2)}\right)$ associated with the factors $\left|\gamma_{n_{l_{s}}}\right|^{-2}$. As

$$
\prod_{\alpha} \prod_{p=1}^{\infty}\left|1-K_{\alpha}^{p}\right|^{-48}>\left(1-\frac{\epsilon_{0}^{\prime}}{g}\right)^{\frac{48}{\frac{\epsilon_{0}^{\prime}}{g}\left(1-\frac{\epsilon_{0}^{\prime}}{g}\right)} \sum_{\alpha}{ }^{\prime}\left|K_{\alpha}\right|}
$$

the primitive-element products are bounded by an exponentially decreasing function of the genus. Similarly,

$$
[\operatorname{det} \operatorname{Im} \tau]^{-13}>(2 \pi)^{13 g}\left[\ln g-\ln \epsilon_{0}+O(1)\right]^{-13 g}
$$

since

$$
\frac{\operatorname{tr}(\operatorname{Im} \tau)}{g}<\frac{1}{2 \pi g} \sum_{n=1}^{g} \sum_{\alpha}(n, n) \frac{\left|\gamma_{\alpha}\right|^{-2}\left|\xi_{1 n}-\xi_{2 n}\right|^{2}}{\left|\xi_{1 n}-V_{\alpha} \xi_{1 n}\right|\left|\xi_{2 n}-V_{\alpha} \xi_{2 n}\right|\left|\xi_{1 n}+\frac{\delta_{\alpha}}{\gamma_{\alpha}}\right|\left|\xi_{2 n}+\frac{\delta_{\alpha}}{\gamma_{\alpha}}\right|}
$$

and the dominant contribution to the second term arises from elements $T_{n_{l}}$ obeying equalities of the form $\left|\xi_{1 n}-T_{n_{l}} \xi_{1 n}\right|=O\left(\frac{1}{\sqrt{g}}\right),\left|\xi_{2 n}-T_{n_{l}} \xi_{1 n}\right|=O(1),\left|\xi_{1 n}+\frac{\delta_{n_{l}}}{\gamma_{n_{l}}}\right|=O(1)$ and $\left|\xi_{2 n}+\frac{\delta_{n_{l}}}{\gamma_{n_{l}}}\right|=0(1)$, together with permutations of these relations. Integration over the multipliers and fixed points in the above range leads to a lower bound

$$
\begin{gathered}
\frac{\left|\xi_{11}^{0}-\xi_{1 g}^{0}\right|^{2}\left|\xi_{21}^{0}-\xi_{1 g}^{0}\right|^{2}}{\left|\xi_{11}^{0}-\xi_{21}^{0}\right|^{2}} \frac{e^{-4 \epsilon_{0}^{\prime}}}{2}(2 \pi)^{16 g-3}\left(\frac{1}{\epsilon_{0}^{2}}-\frac{1}{\epsilon_{0}^{\prime 2}}\right)^{g}\left(\frac{2}{\delta_{0}^{2}}-\frac{2}{\delta_{0}^{\prime 2}}\right)^{g-1}\left(\delta_{2}^{\prime 2}-\delta_{2}^{2}\right)^{g-2} e^{-k(0) g} \\
\cdot \frac{g^{2 g}}{\left[\operatorname{lng}-\ln \epsilon_{0}+O(1)\right]^{13 g}}
\end{gathered}
$$

where $\delta_{2}$ and $\delta_{2}^{\prime}$ represent the inner and outer radii of an annulus containing the isometric circles and $\mathrm{k}(0)$ is a genus-independent constant determined by the primitive-element products

$$
k(0)=52 \lim _{g \rightarrow \infty} \frac{1}{g} \sum_{\alpha}{ }^{\prime}\left|K_{\alpha}\right|
$$

The global SL $(2, \mathbb{C})$ invariance of the Schottky uniformization has been used to fix the values of three points $\xi_{11}, \xi_{21}$ and $\xi_{1 g}$. 
For category (ii), a similar bound is obtained, except that the exponential bound for the primitive-element products is $e^{-k(q) g}$ and the dominant growth is given by

$$
\frac{g^{2(g-g q-q)}}{\left[(1-2 q) \ln g-\ln \epsilon_{0}+O(1)\right]^{13 g}}
$$

For the third category of isometric circles, the integral over the multipliers of the group gives an exponential function of the genus, while the integral over the fixed points is bounded below by a function which increases as $c_{1} c_{2}^{g} g^{g-1}$. A lower bound can also be placed on the determinant factor

$$
\begin{aligned}
(\operatorname{det} \operatorname{Im} \tau)^{-13}>( & 2 \pi)^{13 g}\left[\ln \left(\frac{1}{\epsilon_{0}}\right)\right. \\
& \left.+\frac{1}{g} \sum_{n=1}^{g} \sum_{\alpha}{ }^{(n, n)} \frac{\left|\gamma_{\alpha}\right|^{-2}\left|\xi_{1 n}-\xi_{2 n}\right|^{2}}{\left|\xi_{1 n}+\frac{\delta_{\alpha}}{\gamma_{\alpha}}\right|\left|\xi_{2 n}-\frac{\delta_{\alpha}}{\gamma_{\alpha}}\right|\left|\xi_{1 n}-V_{\alpha} \xi_{1 n}\right|\left|\xi_{2 n}-V_{\alpha} \xi_{2 n}\right|}\right]^{-13 g}
\end{aligned}
$$

The second term can be regarded as the sum of a series involving fundamental generators and their inverses and a series involving elements that are equal to a product of two or more generators. Finiteness of the term may be verified through the convergence of each type of series.

Proposition 1. The remainder term in the lower bound for $[\operatorname{det}(\operatorname{Im} \tau)]^{-13}$ is finite for arbitrary genus.

Proof. As the minimum distance between neighbouring isometric circles in the bounded domain is $\frac{\delta_{0}}{\sqrt{g}}$, the configuration involving the greatest number of circles in a region of fixed area would involve a hexagonal arrangement. Assuming that $\xi_{1 n}$ and $\xi_{2 n}$ lie at the center of the configuration, the isometric circles may be labelled as $I_{T_{n_{l}}}$ and $I_{T_{n_{l}}^{-1}}$, where 1 refers to the level of $I_{T_{n_{l}}}$ with respect to $I_{T_{n}}$. As the number of circles at level 1 is $6(1-1)$, the number 
of levels in a hexagonal configuration is $\left[\frac{1}{2}+\frac{1}{6} \sqrt{9+24 g}\right]$, and since $\left|\xi_{2 n}+\frac{\delta_{n_{l}}}{\gamma_{n_{l}}}\right| \geq(l-1) \frac{\delta_{0}}{\sqrt{g}}$,

$$
\begin{aligned}
& \sum_{n_{l} \neq n} \frac{\left|\gamma_{n_{l}}\right|^{-2}\left|\xi_{1 n}-\xi_{2 n}\right|^{2}}{\left|\xi_{1 n}-T_{n_{l}} \xi_{1 n}\right|\left|\xi_{2 n}-T_{n_{l}} \xi_{2 n}\right|\left|\xi_{1 n}+\frac{\delta_{n_{l}}}{\gamma_{n_{l}}}\right|\left|\xi_{2 n}+\frac{\delta_{n_{l}}}{\gamma_{n_{l}}}\right|} \\
& +\sum_{n_{l} \neq n} \frac{\left|\gamma_{n_{l}}\right|^{-2}\left|\xi_{1 n}-\xi_{2 n}\right|^{2}}{\left|\xi_{1 n}-T_{n_{l}}^{-1} \xi_{1 n}\right|\left|\xi_{2 n}-T_{n_{l}}^{-1} \xi_{2 n}\right|\left|\xi_{1 n}-\frac{\alpha_{n_{l}}}{\gamma_{n_{l}}}\right|\left|\xi_{2 n}-\frac{\alpha_{n_{l}}}{\gamma_{n_{l}}}\right|} \\
& \leq 6 \frac{\delta_{0}^{\prime 4}}{\delta_{0}^{4}} \frac{\epsilon_{0}^{\prime}}{\left[1-\epsilon_{0}^{\prime}\right]^{2}} \sum_{l=2}^{2 \frac{\delta_{0}^{\prime}}{\delta_{0}}}\left[1+\frac{\epsilon_{0}^{\prime \frac{1}{2}}}{1-\epsilon_{0}^{\prime}}\right] \quad[l-1]+\left[\left[6 \frac{\delta_{0}^{\prime 4}}{\delta_{0}^{4}} \frac{\epsilon_{0}^{\prime}}{\left[1-\epsilon_{0}^{\prime}\right]^{2}}\right.\right. \\
& \left.\sum_{2 \frac{\delta_{0}^{\prime}}{\delta_{0}}\left[1+\frac{\epsilon_{0}^{\prime \frac{1}{2}}}{1-\epsilon_{0}^{\prime}}\right]+1}^{\left[\frac{1}{2}+\frac{1}{6} \sqrt{9+24 g}\right]} \frac{[l-1]}{\left.[l-1]\left[l-1-\frac{\delta_{0}^{\prime}}{\delta_{0}}\right]\left[l-1-\frac{\delta_{0}^{\prime}}{\delta_{0}}\left[1+\frac{2 \epsilon_{0}^{\prime \frac{1}{2}}}{1-\epsilon_{0}^{\prime}}\right]\right]\left[l-1-2 \frac{\delta_{0}^{\prime}}{\delta_{0}}\left[1+\frac{\epsilon_{0}^{\prime \frac{1}{2}}}{1-\epsilon_{0}^{\prime}}\right]\right]\right]}\right]
\end{aligned}
$$

When $\xi_{2 n}$ lies in the level $l^{\prime \prime}+1$, it is located at a distance greater than $l^{\prime \prime} \frac{\delta_{0}}{\sqrt{g}}$ from the center of the configuration, and a similar bound is valid with $\left[\frac{1}{2}+\frac{1}{6} \sqrt{9+24 g}\right]$ replaced by $\left[\frac{1}{2}+\frac{1}{6} \sqrt{9+24 g}\right]+l^{\prime \prime}$.

The second type of series, involving a summation over elements $V_{\tilde{\alpha}}=T_{n_{l}} V_{\beta}$, can be bounded even though the location of the isometric circles $I_{V_{\tilde{\alpha}}}$ and $I_{V_{\tilde{\alpha}}^{-1}}$ may not be correlated. The ratio of the inverse square of the radii of the circles $I_{V_{\tilde{\alpha}}}$ and $I_{V_{\tilde{\beta}}}$ obeys the inequality

$$
\left|\frac{\gamma_{\tilde{\alpha}}}{\gamma_{\beta}}\right|^{-2}=\left|\gamma_{n_{l}}\right|^{-2}\left|\frac{\delta_{n_{l}}}{\gamma_{n_{l}}}+\frac{\alpha_{\beta}}{\gamma_{\beta}}\right|^{-2}<\frac{\epsilon_{0}^{\prime}}{\left[1-\epsilon_{0}^{\prime}\right]^{2}} \frac{\delta_{0}^{\prime 2}}{g}\left|\frac{\delta_{n_{l}}}{\gamma_{n_{l}}}+\frac{\alpha_{\beta}}{\gamma_{\beta}}\right|^{-2}
$$

For elements that are products of two generators, or their inverses, it follows that

$$
\begin{aligned}
& \left|\gamma_{\tilde{\alpha}}\right|^{-2}\left|\xi_{1 n}-\xi_{2 n}\right|^{2} \\
& \overline{\left|\xi_{1 n}-V_{\tilde{\alpha}} \xi_{1 n}\right|\left|\xi_{2 n}-V_{\tilde{\alpha}} \xi_{2 n}\right|\left|\xi_{1 n}+\frac{\delta_{\tilde{\alpha}}}{\gamma_{\tilde{\alpha}}}\right|\left|\xi_{2 n}+\frac{\delta_{\tilde{\alpha}}}{\gamma_{\tilde{\alpha}}}\right|} \\
& \leq \frac{\epsilon_{0}^{\prime 2}}{\left[1-\epsilon_{0}^{\prime}\right]^{4}} \frac{\delta_{0}^{\prime 6}}{g^{3}} \frac{\left|\frac{\delta_{n_{l}}}{\gamma_{n_{l}}}+\frac{\alpha_{\beta}}{\gamma_{\beta}}\right|^{-2}}{\left|\xi_{1 n}-V_{\tilde{\alpha}} \xi_{1 n}\right|\left|\xi_{2 n}-V_{\tilde{\alpha}} \xi_{2 n}\right|\left|\xi_{1 n}+\frac{\delta_{\tilde{\alpha}}}{\gamma_{\tilde{\alpha}}}\right|\left|\xi_{2 n}+\frac{\delta_{\tilde{\alpha}}}{\gamma_{\tilde{\alpha}}}\right|}
\end{aligned}
$$

Using this inequality, an upper bound can be obtained for series involving elements $V_{\tilde{\alpha}}$, which are products of two or more fundamental generators. The upper bounds for the 
higher-order terms have the same form as the bound in equation (18), with coefficients $\frac{\delta_{0}^{\prime 2}}{\delta_{0}^{2}}\left[6 \frac{\delta_{0}^{\prime 2}}{\delta_{0}^{2}} \frac{\epsilon_{0}^{\prime}}{\left(1-\epsilon_{0}^{\prime}\right)^{2}}\right]^{j}$, multiplying jth order sums over fractions involving the levels $l^{T_{n_{l_{1}}}^{ \pm 1}}, \ldots, l^{T_{n_{l_{j}}}^{ \pm 1}}$ having leading order behaviour $\left[l^{T_{n_{l_{1}}}^{ \pm 1}}\right]^{r_{1}} \ldots\left[l^{T_{n_{l_{j}}}^{ \pm 1}}\right]^{r_{j}}$ where $\sum_{i=1}^{j} r_{i}=-(j+2)$. This suggests that the entire series containing $\xi_{1 n}$ and $\xi_{2 n}$ is bounded by a geometric series that converges when $6 \frac{\delta_{0}^{\prime 2}}{\delta_{0}^{2}} \frac{\epsilon_{0}^{\prime}}{\left[1-\epsilon_{0}^{\prime}\right]^{2}}<\Delta$, where $\Delta$ is determined by equation (18).

Thus, the lower bound for $[\operatorname{det}(\operatorname{Im} \tau)]^{-13}$ only decreases exponentially. By evaluating the series $\sum_{\alpha}^{\prime}\left|K_{\alpha}\right|=\sum_{\alpha}^{\prime} \frac{\left|\gamma_{\alpha}\right|^{-2}}{\left|\xi_{1 \alpha}+\frac{\delta_{\alpha}}{\gamma_{\alpha}}\right|^{2}}$ for the hexagonal configuration of isometric circles, it may be verified that the primitive- element product $\prod_{\alpha}^{\prime}\left|1-K_{\alpha}\right|^{-1}$ is also bounded below by an exponentially decreasing function of the genus. Combining the lower bounds for the primitive-element products and determinant factor, and the integrals over the multipliers and fixed points, a factorial increase is obtained for the entire integral of the measure (2) in the range for the category (iii).

A procedure similar to the one outlined in this section can be used to set upper bounds for the integrals over each of the three ranges of values for $\left|K_{n}\right|$ and $\left|\xi_{1 n}-\xi_{2 n}\right|, \mathrm{n}=1, \ldots, \mathrm{g}$ $[16]$.

\section{The Fundamental Region of the Symplectic Modular Group}

A rigorous bound can be found for the regularized partition function if the integration region lies within the fundamental domain for the mapping class group. If the coordinates of the Siegel upper half plane are $\left(\tau_{m n}\right)$, where $\operatorname{det}(\operatorname{Im} \tau)>0$, then the restrictions defining the fundamental region of the symplectic modular group [17] are

$$
\begin{aligned}
& \text { (i) } \quad-\frac{1}{2} \leq \operatorname{Re} \tau_{m n} \leq \frac{1}{2} \\
& \text { (ii) } \quad|\operatorname{det}(C \tau+D)| \geq 1 \quad\left(\begin{array}{cc}
A & B \\
C & D
\end{array}\right) \in S p(2 g, \mathbb{Z}) \\
& \text { (iii) } \quad \operatorname{Im} \tau\left[g_{r}\right] \equiv g_{r}^{T}(\operatorname{Im} \tau) g_{r} \geq \operatorname{Im} \tau\left[e_{r}\right] \\
& \quad \text { where } e_{r}=(0, \ldots, 0,1,0, \ldots 0) \text { and } g_{r r}, \ldots, g_{r g} \text { are relatively prime } \\
& \quad(\text { Im } \tau)_{1 r} \geq 0
\end{aligned}
$$

While these conditions can be translated to restrictions on the Schottky coordinates by 
inverting the formula (3) for the period matrix elements, it is simpler to verify that refined limits would lead to the matrix inequalities being satisfied.

An analysis of the real part of the period matrix reveals that the arguments of the fixed point ratios tend to cancel, so that the dominant contribution to $R e \tau_{n n}$ shall be the argument of $K_{n}$. A shift in the argument of $K_{n}$ by $2 \pi$ is equivalent to a shift in $R e \tau_{n n}$. Since the transformation

$$
\tau \rightarrow \tau+\left(\begin{array}{cccc}
0 & & & \\
& \cdot & & \\
& \cdot & \\
& & 0 & \\
& & 1 & \\
& & 0 & \\
& & \cdot & \\
& & & \cdot \\
& & & 0
\end{array}\right)
$$

is symplectic, generalizing the shift $\tau \rightarrow \tau+1$ at one loop, a translation of the argument of $K_{n}$ by $2 \pi$ moves the point in parameter space to a different fundamental region. Since the range for $\arg K_{n}$ will not be reduced significantly by the fixed point ratios, except possibly for the third category of isometric circles, it will be sufficient to integrate, in general, over a interval approximately equal to $[-\pi, \pi]$.

To verify the inequality $|\operatorname{det}(C \tau+D)| \geq 1$, representing the second condition defining the fundamental region, it is preferable to split the determinant

$$
|\operatorname{det}(C \tau+D)|=|\operatorname{det}(\operatorname{Im} \tau)|\left|\operatorname{det}\left(C-i C(\operatorname{Re} \tau)(\operatorname{Im} \tau)^{-1}-i D(\operatorname{Im} \tau)^{-1}\right)\right|
$$

For the first two types of configurations, the diagonal entries of $\operatorname{Im} \tau$ grow as $O(\operatorname{lng})$ and the Minkowski inequality [18] for positive-definite matrices implies

$$
\operatorname{det}(\operatorname{Im} \tau) \geq \frac{(\operatorname{Im} \tau)_{11} \ldots(\operatorname{Im} \tau)_{g g}}{\left.\Gamma\left(\frac{g}{2}+1\right)\right]^{2}}\left(\frac{\pi}{4}\right)^{g}\left(\frac{2}{3}\right)^{g(g-1)} \sim O\left(\frac{\ln g}{g}\right)^{g}\left(\frac{e}{4}\right)^{g}\left(\frac{2}{3}\right)^{g(g-1)}
$$

As the determinant is also equal to the volume of the parallelepiped spanned by the basis vectors $\underline{v}_{1}, \ldots, \underline{v}_{g}$ representing the rows of the matrix, $\left|\underline{v}_{1}\right| \ldots\left|\underline{v}_{g}\right| \sin \theta_{12} \ldots \sin \theta_{1 \ldots g}$, where 
$\theta_{1 \ldots n}$ is the angle from $\underline{v}_{n}$ to the hyperplane spanned by $\underline{v}_{1}, \ldots, \underline{v}_{n-1}$, and since $\sin \theta_{1 \ldots n}=O(1)$ when the off-diagonal entries decrease as $O\left(\frac{1}{\sqrt{g}}\right)$, it follows that $\operatorname{det}(\operatorname{Im} \tau) \geq d(q)^{g} O(l n g)^{g}$ if the off-diagonal elements have a magnitude decreasing with the genus at that rate.

To determine the effect of the off-diagonal elements on the lower bound for the determinant, the following inequalities for eigenvalues of hermitian matrices [19] are relevant. If $\lambda_{1} \leq \lambda_{2} \leq \ldots \leq \lambda_{g}$ are the eigenvalues, $\sigma_{k}^{(n)}$ denotes the sum of the absolute values of off-diagonal elements of the kth row of any principal submatrix of order $\mathrm{n}$ and $h_{k}^{(n)}$ represents the kth diagonal element of the prinicipal submatrix, then

$$
\min _{k}\left[h_{k}^{(g-n+1)}-\sigma_{k}^{(g-n+1)}\right] \leq \lambda_{n} \max _{k}\left[h_{k}^{(n)}+\sigma_{k}^{(n)}\right]
$$

In particular, $\lambda_{1} \leq \min _{n}(\operatorname{Im} \tau)_{n n}$ and $\lambda_{g} \geq \max _{n}(\operatorname{Im} \tau)_{n n}$. Given the period matrix with imaginary part having diagonal entries $\frac{1}{2 \pi} \ln g+O(1)$ and off-diagonal entries $\beta_{m n}$, the following inequalities hold

$$
\begin{aligned}
\lambda_{g} & \geq O(\ln g) \\
\frac{1}{2 \pi} \ln g+O\left(\left|(g-2) \cdot \beta_{m n}\right|\right) & \geq \lambda_{g-1} \geq \frac{1}{2 \pi} \ln g-O\left(\left|\beta_{m n}\right|\right) \\
\frac{1}{2 \pi} \ln g+O\left(\left|(g-3) \cdot \beta_{m n}\right|\right) & \geq \lambda_{g-2} \geq \frac{1}{2 \pi} \ln g-O\left(2\left|\beta_{m n}\right|\right) \\
\ldots & \\
\frac{1}{2 \pi} \ln g & \geq \lambda_{1} \geq \frac{1}{2 \pi} \ln g-O\left(\left|(g-1) \cdot \beta_{m n}\right|\right)
\end{aligned}
$$

To obtain a bound on the lowest eigenvalue of the type $\lambda_{1} \geq O\left(\left|\beta_{m n}\right|\right)$, it is required that $\sigma_{k}^{(j)} \leq \frac{1}{2 \pi} \ln g-O\left(\left|\beta_{m n}\right|\right)$. This would occur if the off-diagonal elements decrease as $O\left(\frac{\ln g}{g}\right)$. Under these conditions, the determinant is greater than $\left(\frac{1}{2 \pi} \ln g\right)^{O(\ln g)}$ is obtained. When the off-diagonal entries are $\mathrm{O}(1)$, a property which holds for the three categories of isometric circles mentioned above, then a lower bound for the determinant can be obtained using the identity $\operatorname{det}\left(\begin{array}{cc}A & B \\ C & D\end{array}\right)=\operatorname{det} A \operatorname{det}\left(D-C A^{-1} B\right)$ and verifying that the determinant does decrease when the diagonal entries are reduced and the offdiagonal entries are increased. If the diagonal elements are chosen to be equal intially, and 
denoted by $\alpha$, and $\beta_{1}<\beta_{m n}<\beta_{2}$, the bound obtained from iteration of the identity is

$$
\begin{aligned}
\alpha^{2-g} & \left(\alpha-\beta_{2}\right)^{g-1}\left(\alpha+\beta_{1}\right)^{3-g}\left(\alpha+\beta_{1}-\beta_{2}\right)^{g-2} \\
& \left(\alpha+2 \beta_{1}\right)^{4-g}\left(\alpha+2 \beta_{1}-\beta_{2}\right)^{g-3} \ldots\left(\alpha+(g-3) \beta_{1}\right)^{-1} \\
& \left(\alpha+(g-3) \beta_{1}-\beta_{2}\right)^{2}\left(\alpha+(g-2) \beta_{1}-\beta_{2}\right)\left(\alpha+(g-1) \beta_{1}\right) \\
& >\left(\alpha-\beta_{2}\right)^{g-1}\left(\alpha+(g-1) \beta_{1}\right) e^{-\frac{\left(\beta_{2}-\beta_{1}\right)}{\beta_{1}} g \ln g \quad \text { as } g \rightarrow \infty} \rightarrow \infty
\end{aligned}
$$

By overlapping the intervals $\left\{\left[\beta_{1}, \beta_{2}\right]|| \beta_{2}-\beta_{1} \mid=\frac{1}{\operatorname{lng}}\right\}$ until $\beta_{1}$ reaches $\frac{\ln g}{g}$, where the theorem about eigenvalues of matrices and their principal submatrices can be used, monotonic growth of the bound with respect to the genus is seen to hold over the interval 0 to $\beta_{m n}=|O(1)|$.

When the diagonal elements of $\operatorname{Im} \tau$ are $O(\ln g)$, the proportion of matrices with $[\operatorname{det}(\operatorname{Im} \tau)]=O(1)$ decreases rapidly with the genus. First, it may be noted that the determinant vanishes when the diagonal elements equal $\frac{1}{2 \pi} \ln g$ and the off-diagonal entries all equal $-\frac{1}{2 \pi} \frac{\ln g}{g-1}$ and is $\mathrm{O}(1)$ only if $\beta_{m n}=-\frac{1}{2 \pi} \frac{\ln g}{g-1}+O\left(\frac{(2 \pi)^{g-1}}{(g-1)(\ln g)^{g-1}}\right)$. If the offdiagonal elements are assumed to be $\mathrm{O}(1)$, then the expression for the determinant defined earlier, $\left|\underline{v}_{1}\right| \ldots\left|\underline{v}_{g}\right| \sin \theta_{12} \ldots \sin \theta_{1 \ldots g}$, will be $\mathrm{O}(1)$ only if $\sin \theta_{12} \ldots \sin \theta_{1 \ldots g}=O\left(\frac{1}{(\ln g)^{g}}\right)$. The analysis may be simplified if it is assumed that the off-diagonal entries are set equal to \pm 1 . Let $n_{2}, \ldots, n_{g}$ denote the number of negative entries in the vector $\underline{v}_{2}, \ldots, \underline{v}_{g}$ respectively. Then

$$
n_{2} \simeq \frac{1}{2} g \sin ^{2} \theta_{12} \quad \ldots \quad n_{g} \simeq \frac{1}{2} g \sin ^{2} \theta_{1 \ldots g}
$$

As there are $\frac{g(g-1)}{2}$ independent off-diagonal entries in the symmetric matrix $I m \tau$, the number of positive entries in the upper triangular part of the matrix can be denoted by $\frac{g(g-1)}{4}+m$, while the number of negative entries would be $\frac{g(g-1)}{4}-m$. Thus,

$$
m \simeq \frac{g}{4}\left[(g-1)-\sin ^{2} \theta_{12}-\ldots-\sin ^{2} \theta_{1 \ldots g}\right]
$$

It would appear that the product constraint could be satisfied when all but one of the angles equals $\frac{\pi}{2}$. However, orthogonality of (g-1) vectors $\underline{v}_{n}$ does not hold when the components of the vectors are \pm 1 . Instead, the signs can be chosen so that only $O(\sqrt{g})$ vectors are 
orthogonal. From the constraint on the product $\sin \theta_{12} \ldots \sin \theta_{1 \ldots g}$, it follows that the minimum value of $\mathrm{m}$ should take the form

$$
\begin{aligned}
m_{\min } & \simeq \frac{g}{4}(g-1-O(\sqrt{g}))\left[1-f_{1}(g) \ldots f_{g-1-O(\sqrt{g})}(g)\right] \\
\prod_{i=1}^{g-1-O(\sqrt{g})} f_{i}(g) & =O\left(\frac{1}{(\ln g)^{g}}\right) \quad f_{i}(g) \rightarrow 0 \quad i=1, \ldots, g-1-O(\sqrt{g}) \text { as } g \rightarrow \infty
\end{aligned}
$$

For large g, it may be assumed that $m \geq \frac{g(g-1)}{8}$. To calculate the proportion of matrices with $\mathrm{m}$ satisfying this inequality, one may note that the signs of the off-diagonal entries of $\operatorname{Im} \tau$ are determined by the arguments of the fixed-point terms and the parameters $\gamma_{\alpha}^{-2}$, which will be randomly distributed in the range $[-\pi, \pi]$ for a large configuration of isometric circles. Thus, the probability of a positive or negative entry will be approximately equal $\frac{1}{2}$, and the computation of the probability becomes identical to the statistical mechanical problem concerning the alignment of spins in a lattice. The number of matrices $\mathrm{N}(\mathrm{m})$ with a given value of $\mathrm{m}$ is $N(0) e^{\frac{-4 m^{2}}{g(g-1)}}$, and the fraction of matrices with $m \geq \frac{g(g-1)}{8}$ is less than $\frac{g(g-1)}{8} e^{-\frac{g(g-1)}{16}}$.

It can be concluded, therefore, that for almost all of the configurations of isometric circles in categories (i) and (ii), $|\operatorname{det}(\operatorname{Im} \tau)|$ will be a rapidly increasing function of the genus. When $\operatorname{det} C \neq 0,\left|\operatorname{det}\left(C-i C(\operatorname{Re} \tau)(\operatorname{Im} \tau)^{-1}-i D(\operatorname{Im} \tau)^{-1}\right)\right|$ will be bounded below by a constant, because $\mathrm{C}$ would have integer entries, whereas the remaining matrices would have rapidly decreasing entries after diagonalization. When $\mathrm{C}=0$, the expression (23) becomes $|\operatorname{det} D| \geq 1$ as $\operatorname{det} D \neq 0$ and $\mathrm{D}$ has integer entries. The determinant condition defining the fundamental region will therefore be satisfied.

For the third category of isometric circles, the diagonal entries of $\operatorname{Im} \tau$ no longer increase logarithmically with the genus, but the determinant inequality will still be valid if $\epsilon_{0}^{\prime}<<1$. In particular, it can be deduced from the general expression (27) that the determinant will be a monotonically increasing function of the genus when

$$
\epsilon_{0}^{\prime}<\exp \left[-B_{0}\left(\max _{n} s_{n n}+\max _{m \neq n} s_{m n}\right)\right]
$$

where $s_{m n}$ is an upper bound for $\sum_{\alpha}(m, n) \ln \left|\frac{\xi_{1 m}-V_{\alpha} \xi_{2 n}}{\xi_{1 m}-V_{\alpha} \xi_{1 n}} \frac{\xi_{2 m}-V_{\alpha} \xi_{1 n}}{\xi_{2 m}-V_{\alpha} \xi_{2 n}}\right|$ and $B_{0}$ is an appropriate constant. 
The third set of conditions defining the fundamental region comprise an infinite set of inequalities for the period matrix inequalities. In the following proposition, it is shown that these inequalities generally reduce to a special class of inequalities, which can be explicitly analyzed.

Proposition 2. For sufficiently large genus, or for a sufficiently small upper limit for the magnitudes of the multipliers, the conditions $g_{r}^{T}(\operatorname{Im} \tau) g_{r} \geq e_{r}^{T}(\operatorname{Im} \tau) e_{r}$ always hold when $(\operatorname{Im} \tau)_{s s} \geq(\operatorname{Im} \tau)_{r r}$ for $s \geq r$.

Proof. When only one entry between $g_{r r}$ and $g_{r g}$ is non-zero, the condition $\operatorname{Im} \tau\left[g_{r}\right] \geq$ $\operatorname{Im} \tau\left[e_{r}\right]$ implies that $g_{r s}(\operatorname{Im} \tau)_{s s} \geq(\operatorname{Im} \tau)_{r r}$, for each $\mathrm{s}=\mathrm{r}, \ldots, \mathrm{g}$, which is valid when $(\operatorname{Im} \tau)_{s s} \geq(\operatorname{Im} \tau)_{r r}$.

When $\left|K_{n}\right|=O(l n g)$ and the contribution from the multipliers dominates the contribution from the fixed points, so that the sequentially ordered eigenvalues of $\operatorname{Im} \tau$ are $\lambda_{n}=O(\operatorname{lng})$ for all $\mathrm{n}=1, \ldots, \mathrm{g}$, the inequality $\frac{(\operatorname{Im} \tau)_{g g}}{\lambda_{1}}<C_{0}$ for some constant $C_{0}$ is satisfied. If $g_{r}^{T} g_{r}>C_{0}$, then $g_{r}^{T}(\operatorname{Im} \tau) g_{r} \geq \lambda_{1} g_{r}^{T} g_{r}>(\operatorname{Im} \tau)_{g g} \geq(\operatorname{Im} \tau)_{r r}$. If $g_{r}^{T} g_{r} \leq C_{0}$, then at most $C_{0} g_{r n}$ are non-zero, including at least one $g_{r s}, s \geq r$. When all of the entries are equal to \pm 1 , the following inequalities will then hold, after appropriate relabelling of the indices.

$$
\begin{aligned}
& (\operatorname{Im} \tau)_{11}+\ldots+(\operatorname{Im} \tau)_{C_{0}-1, C_{0}-1}+(\operatorname{Im} \tau)_{s s} \geq \frac{C_{0}-1}{2 \pi}\left[(1-2 q) \ln g-\ln \epsilon_{0}^{\prime}-\max _{n} s_{n n}\right] \\
& +(\operatorname{Im} \tau)_{r r}, \\
& \text {... } \quad s \geq r \\
& \pm 2(\operatorname{Im} \tau)_{12} \pm \ldots \pm 2(\operatorname{Im} \tau)_{C_{0}-1, s} \geq-\frac{C_{0}\left(C_{0}-1\right)}{2 \pi} \max _{m \neq n} s_{m n}
\end{aligned}
$$

The inequality $g_{r}^{T}(\operatorname{Im} \tau) g_{r} \geq e_{r}^{T}(\operatorname{Im} \tau) e_{r}$ will then be satisfied when $g \geq \epsilon_{0}^{\frac{1}{1-2 q}} \exp \left[\frac{C_{0}}{1-2 q} \max _{m \neq n} s_{m n}+\max _{n} s_{n n}\right]$. Moreover, the derivative of the quadratic form $g_{r}^{T}(\operatorname{Im} \tau) g_{r}$ with respect to $g_{r n}$ is non-negative when $g_{r}^{T} g_{r} \leq C_{0}$ and when the genus is greater than the lower bound just given. Thus, for higher genus, the third set of reduction conditions is equivalent to $(\operatorname{Im} \tau)_{s s} \geq(\operatorname{Im} \tau)_{r r}, s \geq r$. 
The multipliers for isometric circles in the third category have absolute value $\left|K_{n}\right|=O(1)$, so that the diagonal entries of $\operatorname{Im} \tau$ in the inequalities $g_{r}^{T}(\operatorname{Im} \tau) g_{r} \geq$ $e_{r}^{T}(\operatorname{Im} \tau) e_{r}$ are no longer rapidly increasing functions of the genus. However, the eigenvalues are still positive and of the same order, so that the lowest eigenvalue satisfies the inequality $\frac{(\operatorname{Im} \tau)_{g g}}{\lambda_{1}}<C_{0}$ for some constant $C_{0}$. When $g_{r}^{T} g_{r}>C_{0}$, then $\operatorname{Im} \tau\left[g_{r}\right] \geq$ $\operatorname{Im} \tau\left[e_{r}\right]$ immediately follows from $(\operatorname{Im} \tau)_{s s} \geq(\operatorname{Im} \tau)_{r r}, s \geq r$. When $g_{r}^{T} g_{r} \leq C_{0}$, $(\operatorname{Im} \tau)_{s s} \geq(\operatorname{Im} \tau)_{r r}, s \geq r$ implies that the reduction conditions hold if

$$
\epsilon_{0}^{\prime} \leq \exp \left[-C_{0}\left(\max _{n} s_{n n}+\max _{m \neq n} s_{m n}\right)\right]
$$

The inequalities $(\operatorname{Im} \tau)_{s s} \geq(\operatorname{Im} \tau)_{r r}, s \geq r$, lead to constraints of the form $\frac{\left|K_{1}\right|}{\rho_{1}} \geq$ $\frac{\left|K_{2}\right|}{\rho_{2}} \geq \ldots \geq \frac{\left|K_{g}\right|}{\rho_{g}}$ where

$$
\rho_{n}=\prod_{\alpha}{ }^{(n, n)}\left|\frac{\xi_{1 n}-V_{\alpha} \xi_{2 n}}{\xi_{1 n}-V_{\alpha} \xi_{1 n}} \frac{\xi_{2 n}-V_{\alpha} \xi_{1 n}}{\xi_{2 n}-V_{\alpha} \xi_{2 n}}\right|
$$

Denoting $\rho^{-1}=\max \left\{\rho_{1}, \ldots, \rho_{g}\right\}$, the factor $\left(\frac{1}{\epsilon_{0}^{2}}-\frac{1}{\epsilon_{0}^{\prime 2}}\right)^{g}$ in equation $(15)$ is replaced by $\frac{1}{\rho_{1}^{2} \ldots \rho_{g}^{2} g !}\left(\frac{1}{\rho^{-2} \epsilon_{0}^{2}}-\frac{1}{\rho_{1}^{2} \epsilon_{0}^{\prime 2}}\right)^{g}$.

For configurations of isometric circles in category (iii), both terms involving the multipliers and fixed points in $(\operatorname{Im} \tau)_{n n}$ are of order $\mathrm{O}(1)$ so that the inequalities $(\operatorname{Im} \tau)_{s s} \geq$ $(\operatorname{Im} \tau)_{r r}, s \geq r$ may be satisfied by imposing constraints on different sets of parameters, other than the values of $\left|K_{n}\right|, \mathrm{n}=1, \ldots, \mathrm{g}$. It has been shown that restriction of the arguments of $\xi_{1 r}-\xi_{2 r}$ and $K_{r}$ for isometric circles $I_{T_{r}}$ and $I_{T_{r}^{-1}}$ in a local neighbourhood consisting of $\mathrm{J}$ circles can be sufficient to obtain a sequential ordering of $\left\{(\operatorname{Im} \tau)_{r r}\right\}$ for those circles when $\mathrm{J}$ is bounded [16]. This local ordering can be repeated for the entire configuration, reducing the integral over the fixed points by an exponential function of the genus. A representative generator associated with each neighbourhood can then be chosen, and the ordering of the $\left\{(\operatorname{Im} \tau)_{r_{N} r_{N}}\right\}, N=1, \ldots, \frac{g}{J}$ may be achieved either through constraints on the absolute values of the multipliers $K_{r_{N}}$, the distances $\left|\xi_{1 r_{N}}-\xi_{2 r_{N}}\right|$ or both simultaneously. A reduction of the combined integral over the multipliers and fixed points by a factor of order $O\left(\frac{g}{J}\right)$ ! produces a dependence on the genus of $\frac{c_{1} c_{2}^{g} c_{3}^{\frac{g}{J}}}{[\operatorname{det}(\operatorname{Im} \tau)]^{13 g} e^{k\left(\frac{1}{2}\right) g}} g^{g\left(1-\frac{1}{J}\right)}$. 
To satisfy the inequality $(\operatorname{Im} \tau)_{1 n} \geq 0$, suppose that the configuration of isometric circles has the property that $I_{T_{2}^{ \pm 1}}, \ldots, I_{T_{g}^{ \pm 1}}$ are located in a region of the extended complex plane, separated from $I_{T_{1}^{ \pm 1}}$, and that the distances from the fixed points $\xi_{11}$ and $\xi_{21}$ to the group of isometric circles is much greater than the radii of these circles. The restriction on $(\operatorname{Im} \tau)_{1 n}$ implies that

$$
\begin{aligned}
\prod_{\alpha}{ }^{(1, n)} & \left|\frac{\xi_{11}-V_{\alpha} \xi_{2 n}}{\xi_{11}-V_{\alpha} \xi_{1 n}} \frac{\xi_{21}-V_{\alpha} \xi_{1 n}}{\xi_{21}-V_{\alpha} \xi_{2 n}}\right| \\
& =\prod_{\alpha}{ }^{(1, n)}\left|1+\frac{\left(\xi_{21}-\xi_{11}\right)\left(\xi_{1 n}-\xi_{2 n}\right) \gamma_{\alpha}^{-2}}{\left(\xi_{1 n}+\frac{\delta_{\alpha}}{\gamma_{\alpha}}\right)\left(\xi_{2 n}+\frac{\delta_{\alpha}}{\gamma_{\alpha}}\right)\left(\xi_{11}-V_{\alpha} \xi_{1 n}\right)\left(\xi_{21}-V_{\alpha} \xi_{2 n}\right)}\right| \\
& \geq 1
\end{aligned}
$$

Using the freedom to specify the parameters of the generators $T_{n}$, the positions of the fixed points $\xi_{11}$ and $\xi_{21}$ and the arguments of the parameters $\xi_{1 n}-\xi_{2 n}$ and $\gamma_{n}$ can be chosen so that the remainder term in the product in (35) has a phase between $-\frac{\pi}{2}$ and $\frac{\pi}{2}$, giving rise to a fixed-point fraction with absolute value greater than one. As the terms with $V_{\alpha}=T_{m}^{ \pm 1}, m \neq 1, n$ provide the dominant contribution to the product (35), it follows that the period matrix would satisfy the reduction condition for these configurations. The constraints on the arguments reduce the integral over the selected regions in the Schottky group parameter space by an exponential function of the genus.

Having obtained the contributions of the three categories of isometric circles to the integral, it might appear that the continuous parameter $\mathrm{q}$, defining the dependence of the multipliers and fixed points on the genus for the intermediate configurations, could also lead to an infinity. However, it has been demonstrated that the requirement of nonoverlapping of the ranges $\frac{\epsilon_{0}}{g^{1-2 q}} \leq\left|K_{n}\right| \leq \frac{\epsilon_{0}^{\prime}}{g^{1-2 q}}$ forces a selection of approximately $\frac{\ln g}{\ln \left(\frac{\epsilon_{0}^{\prime}}{\epsilon_{0}}\right)}$ evenly spaced values in the interval $\left[0, \frac{1}{2}\right)$. Summing over the allowed values of q gives a lower bound

$$
\frac{O\left(g^{g}\right)}{\left[\ln g-\ln \epsilon_{0}+\ldots\right]^{13 g}}\left[\frac{1}{1-e^{-2(g+1) \ln \left(\frac{\epsilon_{0}^{\prime}}{\epsilon_{0}}\right)}}\right]
$$




\section{Other Configurations of Isometric Circles}

There exist other configurations of isometric circles, besides the three categories listed above, consistent with the condition $\left|\gamma_{n}\right|^{-2} \sim \frac{1}{g}$, but their effect on the bounds for regularized partition function is not significant at large genus. By considering the stereographic projection of the extended complex plane onto the sphere, it can be shown that a disk of area $O\left(g^{2 r-1}\right)$ is projected onto a disk of area $O\left(\frac{1}{g}\right)$ at a distance $O\left(g^{r}\right), r>0$, from the origin. The distances between the fixed points in this configuration are $O\left(g^{r-q}\right), 0 \leq q \leq \frac{1}{2}$, while the integral over $\left|\xi_{2 n}\right|$ grows as $O\left(g^{2 r}\right)$. By equation $(7),\left|K_{n}\right|=O\left(g^{2 q-1}\right)$ so that integration over the multipliers and fixed points and multiplication by the determinant factor $[\operatorname{det}(\operatorname{Im} \tau)]^{-13}$ gives a bound

$$
C(q)^{g} \frac{O\left(g^{(1-2 q) g-2(r+q)}\right)}{\left[(1-2 q) \ln g-\ln \epsilon_{0}+\ldots\right]^{13 g}}
$$

for $0 \leq q<\frac{1}{2}$ and

$$
C\left(\frac{1}{2}\right)^{g} O\left(g^{g-2 r-1}\right)
$$

for $q=\frac{1}{2}$. These bounds have already included the reduction of the integration range because of the conditions defining the fundamental region of the group. Although the range of values of the power $\mathrm{r}$ lie between 0 and $\infty$, non-overlapping of the intervals $\delta_{0} g^{r-q} \leq\left|\xi_{1 n}-\xi_{2 n}\right| \leq \delta_{0}^{\prime} g^{r-q}$ requires that only an evenly spaced discrete set of values of r, $N \frac{\ln \left(\frac{\delta_{0}^{\prime}}{\delta_{0}}\right)}{\operatorname{lng}}, N=0, \ldots, \infty$ be chosen to contribute to the bound for the regularized partition function. Adding all of these contributions gives

$$
C(q)^{g} \frac{O\left(g^{2 g-2 q g-2 q}\right)}{\left[(1-2 q) \ln g-\ln \epsilon_{0}+\ldots\right]^{13 g}}\left[\frac{1}{1-\frac{\delta_{0}^{2}}{\delta_{0}^{\prime 2}}}\right]
$$

for $0 \leq q<\frac{1}{2}$ and

$$
C\left(\frac{1}{2}\right)^{g} O\left(g^{g-1}\right)\left[\frac{1}{1-\frac{\delta_{0}^{2}}{\delta_{0}^{\prime 2}}}\right]
$$

for $q=\frac{1}{2}$. The growth of the estimates with respect to $\mathrm{g}$, associated with the three types of configurations considered in the previous section, is essentially unchanged. 
A larger contribution to the partition function arises from configurations of isometric circles satisfying the condition $\left|\gamma_{n}\right|^{-2} \geq O\left(\frac{1}{g}\right)$. These configurations are allowed, because the cut-off condition on the lengths of closed geodesics does not exclude surfaces with some of the handles having a thickness increasing with the genus and larger than the genus-independent lower bound in the intrinsic metric. This leads to the inclusion of other categories of isometric circles

$$
\begin{aligned}
& \frac{\epsilon_{0}}{g^{1-2 q}} \leq\left|K_{n}\right| \leq \frac{\epsilon_{0}^{\prime}}{g^{1-2 q}} \quad \delta_{0} \leq\left|\xi_{1 n}-\xi_{2 n}\right| \leq \delta_{0}^{\prime} \quad 0<q<\frac{1}{2} \\
& \frac{\epsilon_{0}}{g^{1-2 q^{\prime}}} \leq\left|K_{n}\right| \leq \frac{\epsilon_{0}^{\prime}}{g^{1-2 q^{\prime}}} \quad \frac{\delta_{0}}{g^{q}} \leq\left|\xi_{1 n}-\xi_{2 n}\right| \leq \frac{\delta_{0}^{\prime}}{g^{q}} \quad 0<q<q^{\prime}<\frac{1}{2}
\end{aligned}
$$

There will be a reduction in the integrals over the multipliers and fixed points in these categories, because the limits have a different genus-dependence. The lower bounds for the three types of configurations considered in $\S 2$ and $\S 3$, together with the bounds associated with the additional categories (41) can be combined as the genus-dependence of the magnitudes of the multipliers and fixed-point distances can be assigned to different subsets of the $2 \mathrm{~g}$ isometric circles simultaneously. To obtain a bound corresponding to the inclusion of all of the allowed configurations, it is useful to establish the form of the combinatorial factor [16] multiplying the integral. This factor depends on the distinction between different classes of isometric circles, and in particular, the subdivision of the general categories according to the different values of $\mathrm{q}$ and $q^{\prime}$. Since there are approximately $\frac{1}{2} \frac{(\ln g)^{2}}{\left(\ln \left(\frac{\epsilon_{0}^{\prime}}{\epsilon_{0}}\right)\right)^{2}}$ pairs $\left(q, q^{\prime}\right)$, the number of groupings of multipliers and fixed points is

$$
\frac{\left(g+\frac{1}{2} \frac{(\ln g)^{2}}{\left(\ln \left(\frac{\epsilon_{0}^{\prime}}{\epsilon_{0}}\right)\right)^{2}}-1\right) !}{g !\left(\frac{1}{2} \frac{(\ln g)^{2}}{\left(\ln \left(\frac{\epsilon_{0}^{\prime}}{\epsilon_{0}}\right)\right)^{2}}\right) !} \simeq g\left(\frac{2 g\left(\ln \left(\frac{\epsilon_{0}^{\prime}}{\epsilon_{0}}\right)\right)^{2}}{(\ln g)^{2}}+1\right)^{\frac{1}{2} \frac{(\ln g)^{2}}{\left(\ln \left(\frac{\epsilon_{0}^{\prime}}{\epsilon_{0}}\right)\right)^{2}}}
$$

While this factor increases the lower bounds found in $\S 3$, it is not sufficient to obtain a factorial growth with respect to the genus, as $g^{(l n g)^{2}}$ does not compensate for division by the the logarithmic term $(\ln g)^{13 g}$ in categories (i) and (ii) or the factorial term $\left(\frac{g}{J}\right)$ ! in category (iii). 
The sum of the contributions of the different configurations of isometric circles to the regularized partition function may be compared with the lower bound obtained using the combinatorics of graph theory [1]. Viewing the Riemann surfaces of finite genus as thickened trivalent graphs with branches at the final level intertwined, the number of different pairings of the branches increases at a factorial rate with respect to the genus [20]. While the number of annuli in Teichmuller space associated with the thickening of each of the graphs grows at a factorial rate, the total contribution will be reduced by intersections of the annuli and the condition of integration over a single fundamental region of the symplectic modular group. This may require a study of the modular transformations which would map a Riemann surface, constructed using spheres with 3 punctures (in a manner similar to the Schottky uniformization) [21], to another surface built by joining the spheres at a set of interchanged punctures.

While there is agreement with the results of the graph theory argument regarding the rapid growth of the regularized partition function with respect to the genus, a different conclusion is reached regarding Borel summability of the resulting perturbation series. Unless there are additional contributions to the partition function associated with special configurations of isometric circles, an exact factorial increase is not obtained and the dividing factors $(\ln g)^{13 g}$ and $\left(\frac{g}{J}\right)$ ! cause the Borel-transformed series to be convergent. The lower bounds given in $\S 3$ are relevant, because it may be shown that upper bounds can be found with the similar genus-dependence [16]. Moreover, these results suggest the problem of physically interpreting perturbation series of the type $\sum_{g} \frac{g !}{(\ln g)^{g}}$ and $\sum_{g}\left(\frac{g}{k}\right) !$. Finally, whereas the combinatorics of graph theory appear to lead to a large-order factorial growth, independently of the field or string content of the theory [22], the methods used here may reveal more immediately the higher degree of finiteness of superstring theories $[23]$. 


\section{Growth of the Regularized Partition Function and the Domain of String Perturbation Theory}

The calculation of amplitudes of string scattering processes has led to a definition of the domain of string perturbation theory. In particular, a study of the scattering of four tachyons associated with a class of surfaces uniformized by an infinitely-generated group of Schottky type suggested that the sum over histories should only include a special category of effectively closed manifolds [24]. This class would contain closed surfaces of finite genus and a set of infinite-genus surfaces which can be uniformized by a group of Schottky type and belong to $O_{G}$ by classification theory [25]. This property is consistent with a finitesize interaction region, which would exclude surfaces of infinite extent in the target space, with ideal boundaries of positive linear measure being observed as additional string states. Restricting the domain of string perturbation theory to effectively closed surfaces would appear to be required for consistency of the perturbative expansion of the S-matrix.

The allowed configurations of isometric circles have been selected to be consistent with a genus-independent cut-off on the length of closed geodesics in the intrinsic metric on the Riemann surface [26]. The growth of the bounds for the integrals over each range of values of $\left|K_{n}\right|$ and $\left|\xi_{1 n}-\xi_{2 n}\right|, \mathrm{n}=1, \ldots, \mathrm{g}$, reflects the geometrical characteristics of the string worldsheet. The class of effectively closed surfaces with handles of thickness diminishing at a rate of $\left(\frac{1}{n^{q}}\right), q>\frac{1}{2}$ [24], would be excluded by the cut-off. It might appear that configurations of isometric circles on the extended complex plane with the square of the radii $\left|\gamma_{n}\right|^{-2}$ decreasing as $O\left(\frac{1}{n}\right)$ would be consistent with a genus-independent cut-off on the length of closed geodesics. However, the latter condition has been translated in the Schottky parametrization to the sphere of projection. Since the circles of area $O\left(\frac{1}{n}\right)$ on the complex plane are non-overlapping, they would occupy a region of infinite area. A conformal transformation to a bounded region in the complex plane, would decrease the dependence of the areas to $\left(\frac{1}{n^{2 q}}\right), q>\frac{1}{2}$. Even in the intrinsic metric, which expands areas by a factor of $\mathrm{g}$, the handles would still be accumulating to a point. This example confirms the theorem proven earlier [24] relating surfaces that can be uniformized by groups of Schottky type and those in the class $O_{G}$. For the manifolds considered in this paper, the 
length of the closed geodesics are bounded below and the surfaces of infinite genus could be expanded to be manifolds of infinite extent with boundary. Moreover, the equivalent condition on the radii of the isometric circles $\left|\gamma_{n}\right|^{-2} \sim \frac{1}{g}$ implies that the size would vanish in the infinite-genus limit. These particular surfaces of infinite genus, therefore, could not be uniformized by a Schottky group and belong to $O_{G}$. While these manifolds represent the geometrical limit of surfaces which lie within the domain of string perturbation theory at finite genus, they may be regarded as surfaces beyond the domain of perturbation theory at infinite genus. The source of the divergences at large orders can be identified as a class of surfaces which may be regarded as a non-perturbative effect in the theory. Destabilization of the vacuum by non-perturbative effects has been confirmed, therefore, in an investigation of the regularized path integral, for which the tachyonic divergences arising at each order [27][28] have been eliminated.

The techniques developed in this investigation may be adapted to superstring perturbation theory. Since no regularization of the amplitudes is required, a larger class of surfaces, and in particular, those of type $O_{G}$, can be included in the superstring path integral. To count effectively closed surfaces in the path integral, limits involving a more rapid decrease of $\left|K_{n}\right|$ than that given in $\S 2$ such as

$$
\frac{\epsilon_{0}}{n^{q}}<\left|K_{n}\right|<\frac{\epsilon_{0}^{\prime}}{n^{q}} \quad q \in[1, \infty)
$$

must also be used. However, it has been noted in the proof of perturbative finiteness of the amplitudes at each order [23] that the integral over the multipliers containing the term

$$
\int \frac{d^{2} K_{n}}{\left|K_{n}\right|^{2}\left(\ln \left|K_{n}\right|\right)^{5}}
$$

is finite whenever $\left|K_{n}\right| \rightarrow 0$. Thus, for each value of $\mathrm{q}$, the integral over the multipliers is finite, and it only remains to be determined whether the sum of all of the contributions from the different surfaces is finite. Similar considerations apply to the integrals over the fixed-point variables.

The large-order divergences of bosonic string theory, which have been derived here from the genus-dependence of the multipliers and fixed-point distances, therefore, might 
be eliminated simultaneously with the infrared divergences through the introduction of supersymmetry. This demonstrates the advantage of using the Schottky group formalism, because it provides a deeper insight into the connection between supersymmetry and largeorder perturbation theory.

\section{Conclusion}

Divergences in the scattering amplitudes of the closed bosonic string either arise from the coincidence of vertex operators, the boundary of moduli space or the sum over the genus in the perturbative expansion of the S-matrix. The resolution of the problem of the integration region for the moduli space integral in the Schottky group parametrization has led to rigorous bounds for the regularized vacuum amplitude. The growth of these bounds with respect to the genus, together with the positivity of the terms at each order, indicates a further instablity of the vacuum. The application of these methods to superstring theory would follow from an explicit superstring measure. As the regularization of the moduli space integral through a cut-off on the length of closed geodesics on the surface is no longer

necessary, a larger class of effectively closed surfaces will be included in the evaluation of the superstring path integral.

\section{Acknowledgements.}

This investigation was initiated in Brandeis University, Waltham. I wish to thank Profs. Abdus Salam, Howard Schnitzer and Alberto Verjovsky for their interest and encouragement. Writing of the present version of the manuscript has been completed in the Department of Applied Mathematics and Theoretical Physics, Cambridge, and I would like to thank Prof. S. W. Hawking and Dr. G. W. Gibbons for their hospitality. Financial support was provided by the Atomic Energy Commission, Vienna. 


\section{REFERENCES}

[1] D. Gross and V. Periwal, Phys. Rev. Lett. $\underline{60}$ (1988) 2105 -2108

[2] S. Mandelstam, 'The Interacting String Picture and Functional Integration', Proceedings of the Workshop on Unified String Theories, Institute for Theoretical Physics, Santa Barbara, ed. by M. Green and D. Gross (Singapore: World Scientific, 1986) pp.46 - 102

[3] L. Bers, Bull. Lond. Math. Soc. $\underline{4}(1972) 257$ - 300

[4] J. L. Petersen and J. R. Sidenius, Nucl. Phys. B301 (1988) 247 -266

K. O. Roland, Nucl. Phys. $\underline{B 313}$ (1989) 432 - 446

[5] J. L. Petersen, K. O. Roland and J. R. Sidenius, Phys. Lett. $\underline{B 205}$ (1988) 262 - 266

[6] E. D'Hoker and S. Giddings, Nucl. Phys. B291 (1987) 90 - 112

[7] G. Cristofano, M. Fabbrichesi and K. Roland, Phys. Lett. $\underline{B 236}$ (1990) 159 - 164

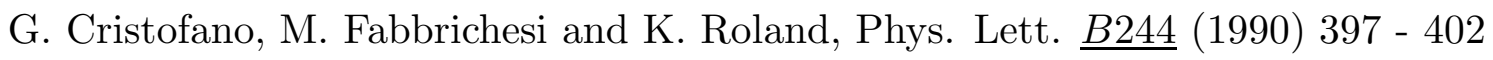

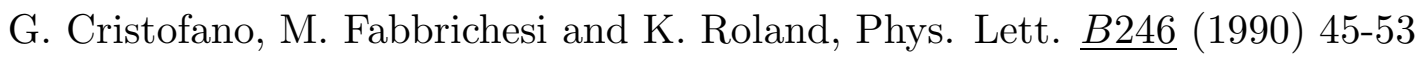

[8] M. Fabbrichesi and R. Iengo, Phys. Lett. $\underline{B 264}$ (1991) 319 - 323

[9] G. Moore, Phys. Lett. $\underline{B 176}$ (1986) 369 - 379

[10] J. Birman, 'The Algebraic Structure of Surface Mapping Class Groups', Discrete Groups and Automorphic Functions, ed. by J. Harvey 
(London: Academic Press, 1977), pp. 163 - 198

[11] S. Nag, The Complex Analytic Theory of Teichmuller Spaces(John Wiley: New York, 1988)

[12] L. Alvarez-Gaume, G. Moore and C. Vafa, Comm. Math. Phys. 106 (1986) 1- 40

[13] A. A. Belavin and V. Knizhnik, Phys. Lett. $\underline{B 168}$ (1986) 201-206

[14] J. Lehner, Discontinuous Groups and Automorphic Functions Mathematical Surveys, Vol. 8 (Providence: American Mathematical Society, 1964)

[15] E. D'Hoker and D. H. Phong, Nucl. Phys. $\underline{B 269}$ (1986) 205 - 234

[16] S. Davis, 'Configurations of Handles and Classification of Divergences in the String Partition Function' (to be published in Class. Quantum Grav., 1994)

[17] C. L. Siegel, Topics in Complex Function Theory, Vol. 3 (Wiley: New York, 1973)

[18] H. Minkowski, Geometrie der Zahlen (New York: Chelsea, 1953)

[19] R. Bhatia, Perturbation Bounds for Matrix Eigenvalues Research Notes in Mathematics, Vol. 162 (Essex:Longman, 1987)

[20] B. Bollobas, J. Lond. Math. Soc. $\underline{26}$ (1982) 201 - 206

[21] C. Vafa, Phys. Lett. $\underline{B 206}$ (1988) 421 - 426

[22] J. Zinn-Justin, Phys. Rep. $\underline{70 C(1981)} 109$ - 167 
[23] N. Berkovits, Nucl. Phys. $\underline{B 408}$ (1993) $43-61$

[24] S. Davis, Class. Quantum Grav. $\underline{6}$ (1989) 1791 - 1803

[25] L. Sario and M. Nakai, Classification Theory of Riemann Surfaces (Berlin: Springer-Verlag, 1970)

[26] S. Davis, Class. Quant. Grav. 7 (1990) 1887 - 1893

[27] P. Nelson, Phys. Rep. $\underline{149 C}$ (1987) 337 - 375

[28] A. A. Tseytlin, Phys. Lett. $\underline{B 208}$ (1988) $221-227$

A. A. Tseytlin, Int. J. Mod. Phys. A. $\underline{4}$ (1989) 1257 - 1318 